\title{
Preoperative prediction of language function by diffusion tensor imaging
}

\author{
C. F. Freyschlag $\cdot$ J. Kerschbaumer $\cdot$ D. Pinggera $\cdot$ \\ T. Bodner • A. E. Grams • C. Thomé
}

Received: 20 January 2017/ Accepted: 13 April 2017/Published online: 4 May 2017

(C) The Author(s) 2017. This article is an open access publication

\begin{abstract}
For surgery of eloquent tumors in language areas, the accepted gold standard is functional mapping through direct cortical stimulation (DCS) in awake patients. Ever since, neuroscientists are searching for reliable noninvasive detection of function in the human brain, with variable success. The potential of diffusion tensor imaging (DTI) in combination with computational cortical parcellation to predict functional areas in language eloquent tumors has not been assessed so far. We present a proof-of-concept report involving awake surgery for a temporodorsal tumor. Postoperatively, the imaging was extensively studied and a predictive value of multimodal MR imaging for the possible extent of resection was analyzed. After resection using DCS, the extent of resection and functional outcome were correlated with the processed imaging. Preoperative imaging of our patient was taken to compute the lesion volume as a seed for tractography (DTI) and combined with a tractography of the entire hemisphere. For better spatial resolution, an elastic image fusion was performed to correct the distortion of DTI data. After subtotal resection and imaging analysis, the status of the superior part of the lesion could be identified and predicted as functional cortex. There was a strong correlation
\end{abstract}

C. F. Freyschlag $(\bowtie) \cdot$ J. Kerschbaumer $\cdot$ D. Pinggera .

C. Thomé

Department of Neurosurgery, Medical University of Innsbruck,

Anichstrasse 35, 6020 Innsbruck, Austria

e-mail: christian.freyschlag@i-med.ac.at

T. Bodner

Department of Neurology, Medical University of Innsbruck,

Anichstrasse 35, 6020 Innsbruck, Austria

\section{A. E. Grams}

Department of Neuroradiology, Medical University of

Innsbruck, Anichstrasse 35, 6020 Innsbruck, Austria between the tumor remnant during surgery and the imaging parameters of DTI connectivity of the eloquent tissue. A combination of complex DTI processing may be able to predict function in a patient suffering eloquent brain tumors and thus allow estimation of extent of resection.

Keywords Diffusion-tensor imaging - Prediction of function · Awake surgery · Eloquent glioma surgery · Functional MRI

\section{Introduction}

Functional resection of brain tumors is key to achieve both, maximized extent of resection and a maintained quality of life for the patients. The gold standard, to maintain neurological function while resection, is the direct stimulation of cortical brain [1-9]. The goal of direct stimulation in awake brain mapping is to optimize the benefit/risk ratio of surgery by avoiding any damage to crucial structures while increasing the extent of resection. To achieve these aims, it is useful to take advantage of modern neuropsychological testing and neuroradiological imaging in combination with direct cerebral mapping, in order to study the individual anatomical and functional organization of the brain. The trend toward less invasive surgery emphasized preoperative planning including different techniques for noninvasive mapping. Functional MRI (fMRI) is available in most centers and could address several brain tasks through the selection of paradigms tested. The sensitivity of fMRI for language investigation is at lowest 59\% [10].

Navigated transcranial magnetic stimulation (nTMS) has shown to obtain a high sensitivity and specificity for prediction of functional areas of the brain [11, 12]. Although this technique has demonstrated safety and 
efficacy-large investments are needed to obtain the technical infrastructure for nTMS. Therefore, we aimed to analyze the potential of diffusion tensor imaging (DTI) and consecutive cortical parcellation to identify localization of language.

Diffusion tensor imaging has shown its reliability in white matter tract visualization, although it represents a rigid mathematical model. In this proof-of-concept study, we analyzed imaging with respect to the functional connectivity of the cortical area involved by a low-grade glioma. The patient underwent awake craniotomy with speech mapping. After resection, the preoperative imaging was analyzed and correlated with the resectable amount of tumor.

\section{Methods}

Our routine MRI low-grade glioma protocol includes T1 w/o contrast, T2, FLAIR, SWI, DWI, MR perfusion, MR spectroscopy and 31phosphorus MR spectroscopy. Within DWI, we routinely perform DTI sequences containing 64 directions.

Imaging was done with a 3.0 T MR scanner (Siemens TRIO, Siemens, Germany). The B-value was set for 1000 . Finally, 38 slices with a thickness of $3 \mathrm{~mm}$ and $0.75 \times 0.75 \mathrm{~mm}$ pixel width were used. Preprocessing was done as follows: eddy current and motion correction (bmatrix rotation) with reference $\mathrm{B} 0$ averaged from multiple B0 and noise-level estimation (PCA-based) and denoising. To enhance spatial resolution, an elastic image fusion algorithm was used to correct the present distortion of DWI. Final image material underwent averaging over repetitions. After preprocessing, the tumor volume was segmentated manually on T2 and FLAIR images. We used standard software for image processing and neuronavigation (iPlan, version 3.2, BrainLAB Inc., Feldkirchen, Germany) including two prototype sections (elastic fusion, fiber tracking) which are currently under licensing procedure.

After segmentation and preprocessing, fiber tracking was done deterministically from the later surgical access zone with a minimum fractional anisotropy (FA) around 0.1 and a minimum fiber length of $10 \mathrm{~mm}$. The resulting connections were then used as source for automatical parcellation of the tumor volume with respect to regions connecting with white or gray matter structures of interest at different points.

Postoperative imaging was compared with the abovementioned processed imaging to determine the extent of resection and a post hoc resection probability analysis (Fig. 1).
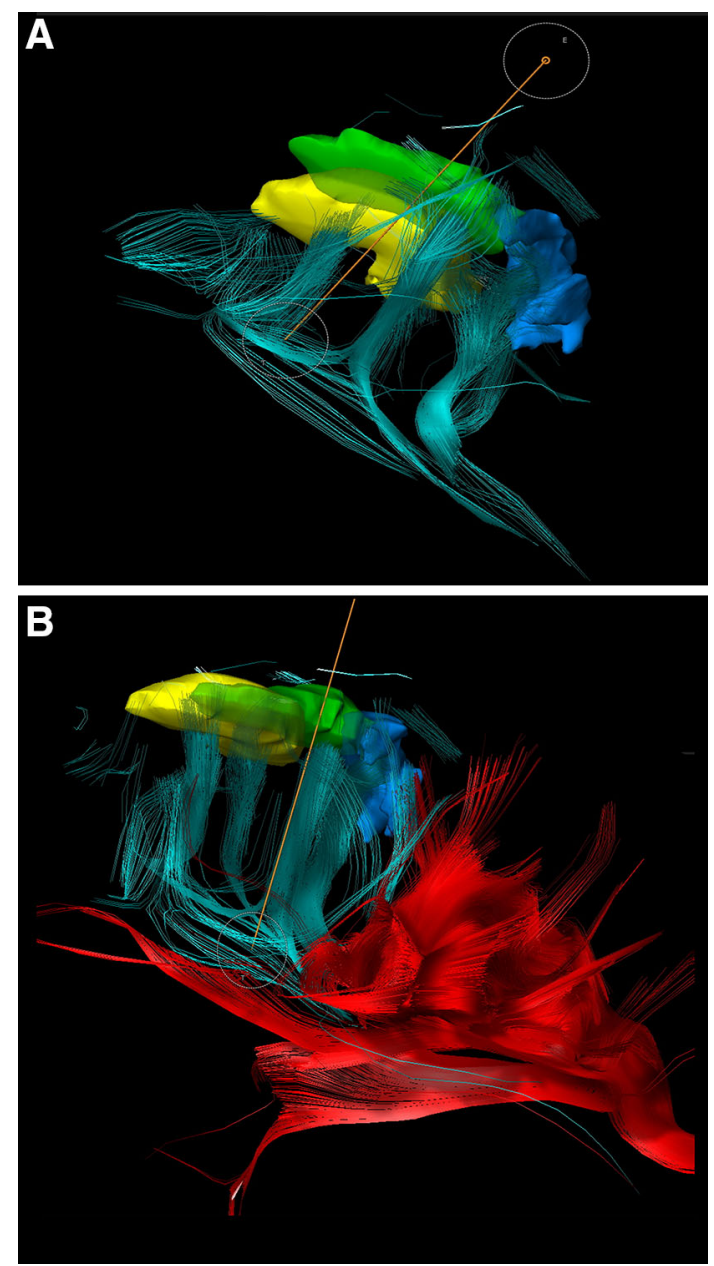

Fig. $13 D$ reconstruction of fiber tracking showing the parcellated cortical areas with corresponding connection fibers. a Fibers between parcellated cortical areas. b Additional tracking of the arcuate fascicle (as a part of SLF) showing the direct connectivity to the cortex segmentated in green. (Color figure online)

\section{Results}

The imaging protocol and processing was applied postoperatively to the imaging of a right-handed female patient with a tumor located in the dorsal left temporal lobe. She showed definite language dominance for the left hemisphere and underwent awake surgery with DCS. Subtotal resection could be achieved due to the eloquence of the most superior part of the tumor, shown in our 3D rendered image (see Fig. 2). While starting a resection attempt of the functional area, the patient developed immediate semantic paraphasia leading to discontinuation of the resection. Intraoperative photography (see Fig. 3) depicts the volume of tumor resected.

Postoperative imaging analysis leads to a similarity index of fiber properties, which was used to determine parcellation boundaries within the total volume of the lesion. The resulting fibers connected to relevant systems in different 


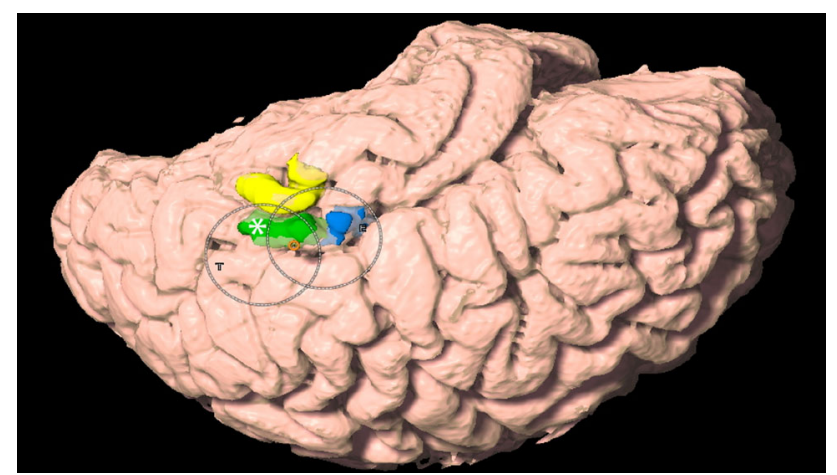

Fig. 2 Whole brain surface rendering showing the parcellated pathological areas divided by their connectivity to the fiber tracts. Green represents the area of direct connection of the lesion to the SLF via arcuate fascicle. (Color figure online)

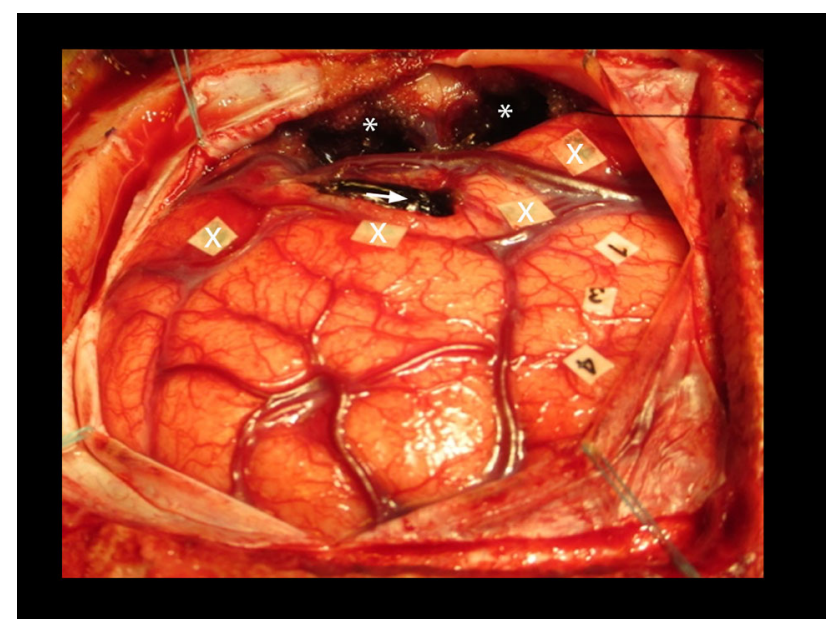

Fig. 3 Intraoperative photograph of the resected tumor (*), sonographic tumor boarders $(X)$ and speech eloquence during direct cortical stimulation (numbers 1, 3 and 4). The arrow marks the area not amenable for resection

ways. The connection of known semantic and phonetic pathways was analyzed, showing that the superior longitudinal fascicle (SLF) connectivity inserted directly in the green area of the tumor (see Fig. 1) via the arcuate fascicle.

\section{Discussion}

The intraoperative direct cortical simulation of speech eloquent brain tissue represents the standard of care in surgery of eloquent lesions $[2,13,14]$. The value of extensive preoperative functional imaging remains debatable, although it is done by many centers.

It is crucial to underline that functional neuroimaging methods suffer various limitations, and are not yet reliable at the individual scale, mainly because they represent biomathematical reconstructions, whose results may change according to the used model [15].
Recently, DTI was also found to be reliable and predictive for functional recovery in patients with ischemic stroke [16], but up to now there has been no report of functional correlation of DTI connectivity with resectability of cortical tissue in low-grade glioma surgery. Interestingly, functional connectivity has been addressed in several studies by the Montpellier group [17], but functional prediction through direct correlation with preoperative DTI connectivity was not yet described. It is obvious that the presence of fiber tracts within the brain white matter does not necessarily represent the functional connectivity of brain areas. According to De Benedictis and Duffau function [2], the hodotopical organization of elaborate does take influence from several streams of information, leading to synchronized cortical activity. Our analysis does not counteract their hypothesis, and it depicts that language function is strongly bound to cortical areas in relation to fiber connectivity.

Fiber tracking is used for access planning and development of resection strategies in supratentorial, perirolandic or thalamic tumors. The mathematical algorithm underlying the technique of tensor tractography is usually a non-functional, rigid algorithm. Given the fact that DTI is subject to massive distortions [18], the integration of distorted tractography to the intraoperative neuronavigation inevitably leads to imprecision.

Overall, these findings pose the question of the necessity that preoperative tumor assessment should be based on connectivity to other gray matter structures (e.g., subcortical and cortical). Future parcellation can easily be based on connectivity to already known tracts (e.g., SLF). It is a matter of ongoing research to combine pre-segmentation and functional results with more functional imaging (e.g., resting-state fMRI).

In our technical proof-of-concept report, we could demonstrate the feasibility of DTI in combination with cortical parcellation. Our study is limited by its retrospective evaluation and the deterministic approach based on the lesion. Prospective trials are ongoing and will further determine the value of tractography in combination with direct cortical stimulation in surgery for eloquent brain tumors. With the present modality, the prediction of function via DTI seems promising.

\section{Conclusion}

Preoperative tractography, based on the parcellation of cortical structures, is nicely reflecting the underlying white matter connectivity. Furthermore, it was possible to validate and even enhance direct intraoperative stimulation under awake conditions. 


\section{Compliance with ethical standards}

Conflict of interest The authors report no conflict of interest for all tools used in the presented manuscript.

Ethical approval For this type of study, formal consent is not required.

Open Access This article is distributed under the terms of the Creative Commons Attribution 4.0 International License (http://crea tivecommons.org/licenses/by/4.0/), which permits unrestricted use, distribution, and reproduction in any medium, provided you give appropriate credit to the original author(s) and the source, provide a link to the Creative Commons license, and indicate if changes were made.

\section{References}

1. Cordier D, Goze C, Schadelin S, Rigau V, Mariani L, Duffau H (2015) A better surgical resectability of WHO grade II gliomas is independent of favorable molecular markers. J Neurooncol 121(1):185-193. doi:10.1007/s11060-014-1623-y

2. De Benedictis A, Duffau H (2011) Brain hodotopy: from esoteric concept to practical surgical applications. Neurosurgery 68(6):1709-1723; discussion 1723. doi:10.1227/NEU.0b013e31 82124690

3. De Witt Hamer PC, Hendriks EJ, Mandonnet E, Barkhof F, Zwinderman AH, Duffau H (2013) Resection probability maps for quality assessment of glioma surgery without brain location bias. PLoS ONE 8(9):e73353. doi:10.1371/journal.pone.0073353

4. Duffau H (2006) New concepts in surgery of WHO grade II gliomas: functional brain mapping, connectionism and plasticity-a review. J Neurooncol 79(1):77-115. doi:10.1007/s11060005-9109-6

5. Duffau H (2009) Surgery of low-grade gliomas: towards a 'functional neurooncology'. Curr Opin Oncol 21(6):543-549. doi:10.1097/CCO.0b013e3283305996

6. Duffau H (2014) Surgical neurooncology is a brain networks surgery: a "connectomic" perspective. World Neurosurg 82(3-4): e405-e407. doi:10.1016/j.wneu.2013.02.051

7. Duffau H, Mandonnet E (2013) The "onco-functional balance" in surgery for diffuse low-grade glioma: integrating the extent of resection with quality of life. Acta Neurochir (Wien) 155(6):951-957. doi:10.1007/s00701-013-1653-9

8. Sarubbo S, De Benedictis A, Merler S, Mandonnet E, Balbi S, Granieri E, Duffau H (2015) Towards a functional atlas of human white matter. Hum Brain Mapp 36(8):3117-3136. doi:10.1002/ hbm. 22832

9. Zemmoura I, Herbet G, Moritz-Gasser S, Duffau H (2015) New insights into the neural network mediating reading processes provided by cortico-subcortical electrical mapping. Hum Brain Mapp 36(6):2215-2230. doi:10.1002/hbm.22766

10. Giussani C, Roux FE, Ojemann J, Sganzerla EP, Pirillo D, Papagno $C$ (2010) Is preoperative functional magnetic resonance imaging reliable for language areas mapping in brain tumor surgery? Review of language functional magnetic resonance imaging and direct cortical stimulation correlation studies. Neurosurgery 66(1):113-120. doi:10.1227/01.NEU.0000360392. 15450.C9
11. Picht T, Krieg SM, Sollmann N, Rosler J, Niraula B, Neuvonen T, Savolainen P, Lioumis P, Makela JP, Deletis V, Meyer B, Vajkoczy P, Ringel F (2013) A comparison of language mapping by preoperative navigated transcranial magnetic stimulation and direct cortical stimulation during awake surgery. Neurosurgery 72(5):808-819. doi:10.1227/NEU.0b013e3182889e01

12. Picht T, Strack V, Schulz J, Zdunczyk A, Frey D, Schmidt S, Vajkoczy P (2012) Assessing the functional status of the motor system in brain tumor patients using transcranial magnetic stimulation. Acta Neurochir (Wien) 154(11):2075-2081. doi:10. 1007/s00701-012-1494-y

13. Benzagmout M, Gatignol P, Duffau H (2007) Resection of World Health Organization Grade II gliomas involving Broca's area: methodological and functional considerations. Neurosurgery 61(4):741-752. doi:10.1227/01.NEU.0000298902.69473.77

14. Duffau H (2013) Brain mapping in tumors: intraoperative or extraoperative? Epilepsia 54(Suppl 9):79-83. doi:10.1111/epi. 12449

15. Duffau H (2014) The dangers of magnetic resonance imaging diffusion tensor tractography in brain surgery. World Neurosurg 81(1):56-58. doi:10.1016/j.wneu.2013.01.116

16. Kumar P, Kathuria P, Nair P, Prasad K (2016) Prediction of upper limb motor recovery after subacute ischemic stroke using diffusion tensor imaging: a systematic review and meta-analysis. J Stroke 18(1):50-59. doi:10.5853/jos.2015.01186

17. Khan OH, Herbet G, Moritz-Gasser S, Duffau H (2014) The role of left inferior fronto-occipital fascicle in verbal perseveration: a brain electrostimulation mapping study. Brain Topogr 27(3):403-411. doi:10.1007/s10548-013-0343-5

18. Kim DJ, Park HJ, Kang KW, Shin YW, Kim JJ, Moon WJ, Chung EC, Kim IY, Kwon JS, Kim SI (2006) How does distortion correction correlate with anisotropic indices? A diffusion tensor imaging study. Magn Reson Imaging 24(10):1369-1376. doi:10.1016/j.mri.2006.07.014

C. F. Freyschlag graduated from Medical University of Innsbruck in 2006 and received his neurosurgical training at the University of Heidelberg, Campus Mannheim, Germany and the Medical University of Innsbruck. Dr. Freyschlag completed fellowships in Montpellier, France and at the Barrow Neurological Institute in Phoenix, Arizona, USA. With being appointed faculty and senior physician at the Department of Neurosurgery he currently serves as head of the neurooncology program. His main research interests are intraoperative Imaging and detection of cortical-subcortical network interaction.

J. Kerschbaumer received his M.D. at the Medical University of Innsbruck in 2010. Focussing on care of neuro-oncological patients in his work, he also conducted clinical trials in glioma patients as well as patients with secondary malignancies of the brain. By integrating multimodal technologies in neurosurgical operating theaters, he started his scientific career on infiltrative processes, interfering with the cortical and subcortical brain network. At the moment, he's working on his $\mathrm{PhD}$ thesis, investigating the brain-tumor border and potential infiltrative mechanisms.

D. Pinggera graduated from Medical University Innsbruck in 2012 and is currently neurosurgical resident. He is part of neurooncology group and his research interest lies in low and high grade glioma, neurocognitive assessment and advanced imaging techniques. Among other things, he takes care of the awake surgery program and pre- and postoperative neurocognitive assessments. 
T. Bodner is a clinical neuropsychologist. Her graduated in clinical psychology and health psychology. His main research interests are neuropsychology in epilepsy and neuropsychological changes in the elderly (e.g. dementia). He is board member of the Austrian Neuropsychological Society (GNPÖ) and managing member of the Federation of the European Neuropsychology societies (FESN).

A. E. Grams is Assistant Professor for Neuroradiology. One of her main research topics is highfield and ultra-highfield Magnetic Resonance imaging, especially the application of 31P Magnetic Resonance Spectroscopy in various diseases of the brain. Her other main research field is the clinical use of Dual Energy computed tomography, especially in stroke patients.
C. Thomé completed his medical education at the Ludwig-Maximilian-Universitaet Munich, Germany and at Stanford University School of Medicine, Stanford/CA, USA followed by his residency in the Department of Neurosurgery, University of Heidelberg, Campus Mannheim (Chairman: Prof. Dr. P. Schmiedek) (19952001). After serving as Professor and Vice-Chairman at the Dept. of Neurosurgery, University of Heidelberg, Campus Mannheim from 2007 to 2009, he was appointed Full Professor and Chairman of the Dept. of Neurosurgery, Medical University Innsbruck, Austria in 2010. Dr. Thomé's surgical specialties are: cerebrovascular surgery, skull base surgery and complex spine surgery, and his main research fields are: cerebrovascular diseases, neuromonitoring in brain injury (TBI, SAH) as well as evidence-based and minimally invasive spine surgery and regenerative medicine in spinal disease. 